\title{
Minimum Alveolar Concentration for Blunting Adrenergic Responses (MAC-BAR) of Sevoflurane in Dogs
}

\author{
Kazuto YAMASHITA ${ }^{1) *}$, Erika FURUKAWA ${ }^{1)}$, Takaharu ITAMI ${ }^{1)}$, Tomohito ISHIZUKA ${ }^{1)}$, Jun TAMURA ${ }^{1)}$ and \\ Kenjirou MIYOSHI ${ }^{1)}$ \\ ${ }^{1)}$ Department of Small Animal Clinical Sciences, School of Veterinary Medicine, Rakuno Gakuen University, Ebetsu, Hokkaido \\ 069-8501, Japan
}

(Received 7 June 2011/Accepted 11 November 2011/Published online in J-STAGE 25 November 2011)

\begin{abstract}
It is well known that heart rate or arterial blood pressure may increase in response to surgical stimulation despite the absence of a purposeful movement. However, there is limited information regarding anesthetic requirement for blunting adrenergic response in dogs. This study was designed to compare the minimum alveolar concentrations of sevoflurane required to prevent autonomic response (MAC-BAR) and purposeful movement (MAC) in dogs. Sevoflurane MAC-BAR and MAC were determined in 5 beagle dogs by judging dogs' response to a noxious electrical stimulus applied to the gingiva. The sevoflurane MAC-BAR was significantly higher than MAC $(3.33 \pm 0.48$ vs $2.10 \pm 0.28 \%, P=0.005)$. These results suggested that autonomic responses occurred at sevoflurane anesthetic concentrations at which purposeful movements were absent.
\end{abstract}

KEY WORDS: anesthesia, canine, MAC-BAR, sevoflurane.

doi: 10.1292/jvms.11-0274; J. Vet. Med. Sci. 74(4): 507-511, 2012

Sevoflurane is a volatile anesthetic agent with a relatively low blood/gas solubility coefficient resulting in rapid induction and recovery from anesthesia [23]. During the last decade, clinical use of sevoflurane has been spreading in veterinary species such as horses [8, 12, 18, 27], cats $[13]$ and dogs $[5,6]$. Sevoflurane is minimally metabolized and easily cleared in animals; however, it should be remembered that sevoflurane causes dose-dependent hypotension, hypoventilation, impaired cardiac contractility and hypothermia [16]. Because of these side effects, sevoflurane must be carefully titrated, and vigilant monitoring should be employed to avoid excessive anesthetic depth.

Assessment of adequate level of anesthesia traditionally has relied on a patient's movement, hemodynamic response, or both to a surgical stimulus. MAC has been defined as "the minimum alveolar concentration of anesthetic at 1 atmosphere that produces immobility in 50\% of those animals exposed to a noxious stimulus" [9]. As a guideline, most patients require approximately 1.2 to 1.4 MAC for an adequate level of surgical anesthesia [23]. On the other hand, it is well known that heart rate or arterial blood pressure may increase in response to surgical stimulation despite the absence of a motor response. Current evidence suggests that volatile inhalant anesthetic agents act primarily within the spinal cord to produce some of their desired clinical effects, including decreased immobility $[4,19]$ and their predominant site of action within

*Correspondence to: Yamashita, K., Department of Small Animal Clinical Sciences, School of Veterinary Medicine, Rakuno Gakuen University, Ebetsu, Hokkaido 069-8501, Japan.

e-mail: yamasita@rakuno.ac.jp

(C)2012 The Japanese Society of Veterinary Science the spinal cord for immobility is the ventral rather than the dorsal horn $[2,10]$. Autonomic responses such as increases in heart rate and arterial blood pressure in response to surgical stimulation are mediated primarily by subcortical centers (hypothalamus, brain stem and spinal cord). Minimum alveolar concentration of blunting adrenergic responses (MAC-BAR) is the minimum inhalant anesthetic concentration that prevents an autonomic response to a noxious stimulus [20] and a useful measure of the effects of an anesthetic on autonomic pathways in the spinal cord and brain stem [22].

MAC-BAR may provide an indirect assessment of the level of analgesia [22]. March et al. [15] demonstrated that MAC-BAR was comparable with alveolar concentration of inhalation anesthetic necessary to prevent cortical arousal in cats anesthetized with isoflurane. Roizen et al. [20] reported that a greater alveolar concentration of inhalation anesthetics was necessary to prevent autonomic adrenergic responses to surgical stimulus (i.e., MAC-BAR) compared with necessary to just prevent movements (i.e., MAC). A ratio of MAC-BAR/MAC for a volatile anesthetic agent should provide a valuable piece of information to achieve an adequate level of surgical anesthesia that prevents autonomic adrenergic responses and movements in animals. However, there are few animal studies investigating a ratio of MAC-BAR/MAC and the results of previous studies are quite disparate between volatile anesthetic agents and species $[1,15,21]$. The purpose of present study was to determine and compare the MAC-BAR and MAC in dogs anesthetized with sevoflurane. The authors hypothesized that the sevoflurane MAC-BAR value would be higher than the sevoflurane MAC value in this species.

Five intact female beagle dogs, 9 to 11 years of age (9.6 
\pm 0.9 [mean \pm standard deviation] years old) and weighing from 8.6 to $13.0 \mathrm{~kg}(11.4 \pm 1.8 \mathrm{~kg})$, were anesthetized with sevoflurane on two occasions with a minimum 14-day washout period to determine sevoflurane MAC and MACBAR. The dogs were judged to be in good to excellent health based upon a physical examination, complete blood cell count and chemical analysis. Food and water were withheld from the dogs for $12 \mathrm{hr}$ before the experiment. The dogs were owned by the university and cared for according to the principles of the "Guide for the Care and Use of Laboratory Animals" prepared by Rakuno Gakuen University. The Animal Care and Use Committee of Rakuno Gakuen University approved the study.

Anesthesia was induced by mask induction using sevoflurane (SevoFlo, Dainippon Sumitomo Pharma, Osaka, Japan) in oxygen. All dogs were orotracheally intubated with an endotracheal tube and anesthetized with oxygensevoflurane anesthesia in left lateral recumbency. Once the dogs were positioned in lateral recumbency, a 22-gauge catheter was placed in the dorsal pedal artery. Arterial blood pressure was directly measured by connecting this catheter to a pressure transducer (CDX-A90, Cobe Laboratories, Tokyo, Japan) placed and zeroed at the level of the mid-sternum. Arterial blood samples were also anaerobically collected from this catheter into a syringe containing heparin to analyze the partial pressures of arterial $\mathrm{O}_{2}$ $\left(\mathrm{PaO}_{2}\right)$ and $\mathrm{CO}_{2}\left(\mathrm{PaCO}_{2}\right)$. During anesthesia, $\mathrm{PaCO}_{2}$ was maintained between 35 and $40 \mathrm{mmHg}$ by intermittent positive pressure ventilation (IPPV). All dogs were administered lactated Ringer's solution at a rate of $10 \mathrm{ml} / \mathrm{kg} / \mathrm{hr}$ intravenously through a 22-gauge catheter placed in the right cephalic vein. Esophageal temperature was maintained between 37.5 and $38.5^{\circ} \mathrm{C}$ using a heating pad and a warm air blanket in all dogs.

Heart rate (beats/min), lead II of the electrocardiogram and arterial blood pressure was recorded by a multi-parameter anesthetic monitoring system (DS-5300, Fukuda Denshi Co., Tokyo, Japan). $\mathrm{PaO}_{2}$ and $\mathrm{PaCO}_{2}$ were determined with a blood gas analyzer (GEM Premier 3000, IL Japan, Tokyo, Japan). Esophageal temperature $\left({ }^{\circ} \mathrm{C}\right)$, respiratory rate (breaths/min) and end-tidal concentration of sevoflurane (ETSEV;\%) were monitored using a veterinary patient monitoring system (BP-508V, Omron Colin Co., Ltd., Tokyo, Japan). The esophageal temperature was measured using an electric thermometer probe placed orally into the thoracic esophagus. A commercially available adaptor (Airway adaptor L-shape, Omron Colin Co.) modified with an 8-Fr feeding tube (Safeed feeding tube, Terumo, Tokyo, Japan) was placed at the Y-piece of the breathing circuit. The feeding tube passed through the endotracheal tube so that its tip rested in the thoracic portion of the trachea. Gas samples were drawn from the proximal end of the endotracheal tube using the feeding tube at a rate of $200 \mathrm{ml} / \mathrm{min}$. A side-stream capnograph and anesthetic agent monitor were used to determine respiratory rate and ETSEV. The anesthetic agent monitor was calibrated immediately prior to each sevoflurane MAC and MAC-BAR determination.

The MAC and MAC-BAR of sevoflurane were deter- mined by judging the dogs' response to a noxious electrical stimulus $(50 \mathrm{~V}, 50 \mathrm{~Hz}, 10 \mathrm{msec})$ [14] applied to their right upper gingival. After the dogs were allowed to equilibrate for $60 \mathrm{~min}$ at ETSEV 2.8 to $3.0 \%$, the electrical stimulus was applied for $10 \mathrm{sec}$ using an electrical stimulator (SEN3301, Nihon Kohden Co., Tokyo, Japan). Positive response to the electrical stimulus for MAC determination was fixed a purposeful movement defined as substantial movement of the head or extremities and did not include coughing, chewing, swallowing or increasing respiratory effort [26, 28]. Positive response for MAC-BAR determination was fixed an increase of either heart rate or mean arterial blood pressure (MABP) over $15 \%$ above the value recorded at $1 \mathrm{~min}$ before applying the electrical stimulus [15]. When the dog exhibited the positive responses, the ETSEV was increased by 10 to $20 \%$, and the dog was retested after 20 min of re-equilibration. When the dog did not exhibit the positive responses, the ETSEV was decreased by 10 to $20 \%$, and the dog was retested after $20 \mathrm{~min}$ of re-equilibration. The MAC and MAC-BAR were determined as the mean of the ETSEV at which the dog did not demonstrate positive responses and next lower concentration tested (i.e., the highest concentration at which the dog demonstrated positive responses to the electrical stimulus). The MAC and MAC-BAR for each dog were determined in triplicate by the same person (K.Y.). Cardio-respiratory data were collected $1 \mathrm{~min}$ before applying the electrical stimulus. The data were reported as means \pm standard deviation and analyzed by use of the paired $t$-test. The level of significance was set at $P<0.05$.

It took $228.0 \pm 27.1$ and $240.0 \pm 58.5 \mathrm{~min}$ after induction of anesthesia to obtain the triplicate data for sevoflurane MAC and MAC-BAR in the dogs, respectively. There was no significant difference in the duration required to determine MAC-BAR and MAC $(P=0.688)$. Sevoflurane MAC$\mathrm{BAR}$ and $\mathrm{MAC}$ was $3.33 \pm 0.48$ and $2.10 \pm 0.28 \%$, respectively. The sevoflurane MAC-BAR was significantly higher than the sevoflurane $\mathrm{MAC}(P=0.005)$ and the ratio of the MAC-BAR value to the MAC value (MAC-BAR/MAC) was $1.61 \pm 0.30$ (i.e., 1.61 MAC) (Table 1).

Esophageal temperature and cardio-respiratory parameters recorded immediately prior to the determination of MAC-BAR and MAC were summarized in Table 2. Throughout the present study, normothermia was achieved by warming with the heating pad and air blanket in all dogs. Good oxygenation and eucapnia were also achieved by IPPV. Statistically significant differences were detected in the esophageal temperature $(P=0.0002)$, heart rate $(P=0.0007), \mathrm{PaO}_{2}(P=0.0350)$ and $\mathrm{PaCO}_{2}(P=0.0001)$. MABP recorded during MAC-BAR determination was significantly lower and close to the clinically lower limit (60 $\mathrm{mmHg}$ ) compared to that recorded during MAC determination $(P=0.0015)$.

In the present study, the sevoflurane MAC-BAR for the dogs was $3.33 \pm 0.48 \%$, which is somewhat higher than the sevoflurane MAC-BAR (2.77\%) for intact male mixedbreed dogs recently reported by Love et al. [14]. Love et al. [14] mentioned that their sevoflurane MAC-BAR was lower 
Table 1. Minimum alveolar concentration for blunting adrenargic response (MAC-BAR) and preventing purposeful movement (MAC) of sevoflurane, and the ratio of MACBAR to MAC in 5 female beagle dogs

\begin{tabular}{ccccc}
\hline Dogs & Age (years) & MAC-BAR (\%) & MAC (\%) & MAC-BAR/MAC \\
\hline No. 1 & 9 & 3.78 & 1.82 & 2.08 \\
No. 2 & 11 & 2.95 & 1.80 & 1.64 \\
No. 3 & 10 & 2.68 & 2.13 & 1.26 \\
No. 4 & 9 & 3.57 & 2.37 & 1.51 \\
No. 5 & 9 & 3.65 & 2.37 & 1.54 \\
\hline Mean \pm SD & $9.6 \pm 0.9$ & $3.33 \pm 0.48^{*}$ & $2.10 \pm 0.28$ & $1.61 \pm 0.30$ \\
\hline
\end{tabular}

Significantly different from the MAC values $(P=0.005)$.

Table 2. Esophageal temperature, heart rate, mean arterial blood pressure (MABP), respiratory rate and partial pressures of arterial oxygen $\left(\mathrm{PaO}_{2}\right)$ and carbon dioxide $\left(\mathrm{PaCO}_{2}\right)$ at the determination of MAC-BAR and MAC of sevoflurane in dogs

\begin{tabular}{lcc}
\hline & MAC-BAR & MAC \\
\hline Esophageal temperature $\left({ }^{\circ} \mathrm{C}\right)$ & $37.8 \pm 0.3^{*}$ & $38.0 \pm 0.2$ \\
Heart rate $(\mathrm{bpm})$ & $116.9 \pm 17.5^{*}$ & $108.7 \pm 18.6$ \\
$\mathrm{MABP}(\mathrm{mmHg})$ & $65.7 \pm 6.1^{*}$ & $73.9 \pm 11.0$ \\
Respiratory rate $(\mathrm{bpm})$ & $13.4 \pm 5.4$ & $13.0 \pm 3.3$ \\
$\mathrm{PaO}_{2}(\mathrm{mmHg})$ & $530.5 \pm 25.0^{*}$ & $559.6 \pm 30.1$ \\
$\mathrm{PaCO}_{2}(\mathrm{mmHg})$ & $39.8 \pm 2.7^{*}$ & $34.3 \pm 3.0$ \\
\hline
\end{tabular}

Data are expressed as means \pm standard deviation for 15 observations from 5 dogs. Data from 3 observations recorded immediately prior to electrical stimulation that produced changes in response to stimulation were obtained from each dog. $* P<0.05$ : significantly different from the values at the detemination of MAC of sevoflurane.

than expected value based on a previous canine study using the same methodology, documented a sevoflurane MACBAR of $3.21 \%$ and a MAC of $1.93 \%$ (Wilson et al. 2008 . Determination of MAC and MAC derivatives for isoflurane and sevoflurane. Proceedings of the American College of Veterinary Anesthesiologists, Scottdale, AZ, pp.4-5), indicating the ratio of MAC-BAR/MAC was $1.71 \mathrm{MAC}$. These results from the canine study by Wilson et al. are quite similar to those in the present study. A composition of gender in dogs is a great difference in experimental protocol between the present study (all intact female dogs) and the study reported by Love et al. [14] (all intact male dogs). Gender difference in the MAC-BAR was supposed in human patients $[11,24]$. Further investigation is necessary to confirm the effect of gender on the MAC-BAR in dogs.

In the present study, the sevoflurane MAC determined in the dogs with electrical stimulation was $2.10 \pm 0.28 \%$, which is somewhat less than those determined in 6 beagle dogs aged 2 years with a tail clamp method $(2.39 \pm 0.26 \%)$ [28]. Differences in study design, individual animal and age may account for variability in MAC results. It is reported that sevoflurane MAC reduced with increasing age of dogs [26], similar to humans [17]. The age of dogs used in the present study were 9 to 11 years, therefore, we surmised that the effect of aging might be a major factor lowering the sevoflurane MAC value. On the other hand, the effect of aging on MAC-BAR has not been investigated in dogs. Further investigation is necessary to confirm the effect of aging on the sevofulane MAC-BAR value in dogs.
Essential features of general anesthesia for surgery include reversible unconsciousness, muscle relaxation, amnesia, and analgesia. In addition, hemodynamic stability during the peri-operative period is desirable because increases in heart rate and blood pressure reflect activation of the neuroendcrine stress response [1]. This sympathetically driven response can be disadvantageous when inappropriately activated during the peri-operative period [25]. The excess and/or prolonged neuroendocrine stress response induced by surgical manipulation are related to adverse effects of surgery such as protein catabolic response, limitation the availability of glucose to tissues, depression of the immune system and prolongation of wound healing and tissue repair [25]. Prevention of the cardiovascular reaction to noxious stimuli may indicate protection from these deleterious effects of the stress response to anesthesia and surgery [20]. Therefore, an "adequate" level of anesthesia should be provided with blunting the autonomic response besides immobilization of patients.

The ratio of MAC-BAR/MAC may reflect analgesic property, cardiovascular depressant effect and/or motoneuron depressant effect of each volatile anesthesic agent. The value of ratio of MAC-BAR/MAC is quite disparate between volatile anesthetic agents and species. It is reported that the ratio of MAC-BAR/MAC was 2.24 MAC for sevoflurane in human [11], 2.64 MAC for isoflurane in goats [1], 1.11 MAC for isoflurane in cats [15] and 1.48 MAC for halothane in cats [21]. Differences in study design, species, age, and volatile anesthetic agents may account for variability 
in MAC-BAR and MAC results. Volatile anesthetic agents may suppress hemodynamic response to noxious stimulation by their analgesic properties with or without direct action on cardiovascular function [3] and may affect cardiovascular responses to noxious stimulation via their actions in brain [1]. As predicted, the sevoflurane MAC-BAR value was significantly higher than the MAC value and mean ratio of MAC-BAR/MAC was 1.61 MAC for sevoflurane in dogs. Our results demonstrated that a greater alveolar concentration of sevoflurane was indispensable for preventing adrenergic responses to stimulus compared to the concentration required for preventing movement in dogs. However, we cannot exclude any influence of direct cardiovascular suppressive effect produced by sevoflurane on the determination of MAC-BAR in the present study. Therefore, the true sevoflurane MAC-BAR may be greater than the MAC-BAR determined in the present study. Direct measure of autonomic activities may be necessary for the determination of MAC-BAR to exclude the direct suppressive action of volatile anesthetics on cardiovascular function.

Mutoh et al. [16] reported that arterial blood pressure and systemic vascular resistance decreased in dose-dependent manner in dogs anesthetized with 1.0, 1.5 and 2.0 MAC of sevoflurane. In the present study, heart rate and mean arterial blood pressure were within the normal values for dogs during anesthesia; however, a mild but clinically meaningful decrease in arterial blood pressure was observed during sevoflurane MAC-BAR determination. Because the cardiovascular depression produced by sevoflurane is dosedependent in dogs, the concentrations required for blocking the autonomic response can generate considerable cardiovascular instability. Analgesic drugs will reduce the MACBAR for volatile anesthetic agents [7, 14]. Therefore, it may be preferable to combine sevoflurane with adjunctive analgesics, such as opioids, non-steroidal anti-inflammatory drugs, ketamine, and local blocks using local anesthetics in order to diminish the hemodynamic consequences of high doses of sevoflurane by decreasing the MAC-BAR to clinically relevant concentrations.

In conclusion, the sevoflurane MAC-BAR was significantly higher than MAC in dogs. The autonomic responses occurred at sevoflurane anesthetic concentrations at which purposeful movements was absent. It may be preferable to combine sevoflurane with adjunctive analgesics to achieve the "adequate" level of anesthesia with clinically relevant sevoflurane concentrations in dogs.

\section{REFERENCES}

1. Antognini, J. F. and Berg, K. 1995. Cardiovascular responses to noxious stimuli during isoflurane anesthesia are minimally affected by anesthetic action in the brain. Anesth. Analg. 81: 843-848. [Medline]

2. Antognini, J. F. and Carstens, E. 1999. Increasing isoflurane from 0.9 to 1.1 minimum alveolar concentration minimally affects dorsal horn cell responses to noxious stimulation. Anesthesiology 90: 208-214. [Medline] [CrossRef]

3. Antognini, J. F. and Carstens, E. 2002. In vivo characterization of clinical anaesthesia and its components. Br. J. Anaesth.
89: 156-166. [Medline] [CrossRef]

4. Antognini, J. F. and Schwartz, K. 1993. Exaggerated anesthetic requirements in the preferentially anesthetized brain. Anesthesiology 79: 1244-1249. [Medline] [CrossRef]

5. Bennett, R. C., Fancy, S. P., Walsh, C. M., Brown, A. J. and Taylor, P. M. 2008. Comparison of sevoflurane and isoflurane in dogs anaesthetized for clinical surgical or diagnostic procedures. J. Small Anim. Pract. 49: 392-397. [Medline] [CrossRef]

6. Branson, K. R., Quandt, J. E., Martinez, E. A., Carroll, G. L., Trim, C. M., Dodam, J. R., Hartsfield, S. M., Matthews, N. S., Mackenthun, A. and Beleau, M. H. 2001. A multisite case report on the clinical use of sevoflurane in dogs. J. Am. Anim. Hosp. Assoc. 37: 420-432. [Medline]

7. Daniel, M., Weiskopf, R. B., Noorani, M. and Eger, E. I. 2nd. 1998. Fentanyl augments the blockade of the sympathetic response to incision (MAC-BAR) produced by desflurae and isoflurane: desflurane and isoflurane MAC-BAR without or with fentanyl. Anesthesiology 88: 43-49. [Medline] [CrossRef]

8. Driessen, B., Nann, L., Benton, R. and Boston, R. 2006. Differences in need for hemodynamic support in horses anesthetized with sevoflurane as compared to isoflurane. Vet. Anaesth. Analg. 33: 356-367. [Medline] [CrossRef]

9. Eger, E. I. 2nd., Saidman, L. J. and Brandstar, B. 1965. Minimum alveolar anesthetic concentration: a standard of anesthetic potency. Anesthesiology 26: 756-763. [Medline] [CrossRef]

10. Jinks, S. L., Bravo, M. and Hayes, S. G. 2008. Volatile anesthetic effects on midbrain-elicited locomotion suggest that the locomotor network in the ventral spinal cord is the primary site for immobility. Anesthesiology 108: 1016-1024. [Medline] [CrossRef]

11. Katoh, T., Kobayashi, S., Suzuki, A., Iwamoto, T., Bito, H. and Ikeda, K. 1999. The effect of fentanyl on sevoflurane requirements for somatic and sympathetic responses to surgical incision. Anesthesiology 90: 398-405. [Medline] [CrossRef]

12. Leece, E. A., Corletto, F. and Brearley, J. C. 2008. A comparison of recovery times and characteristics with sevoflurane and isoflurane anaesthesia in horses undergoing magnetic resonance imaging. Vet. Anaesth. Analg. 35: 383-391. [Medline] [CrossRef]

13. Lerche, P., Muir, W. W. and Grubb, T. L. 2002. Mask induction of anaesthesia with isoflurane or sevoflurane in premedicated cats. J. Small Anim. Pract. 43: 12-15. [Medline] [CrossRef]

14. Love, L., Egger, C., Rohrbach, B., Cox, S., Hobbs, M. and Doherty, T. 2011. The effect of ketamine on the MAC (BAR) of sevoflurane in dogs. Vet. Anaesth. Analg. 38: 292-300. [Medline] [CrossRef]

15. March, P. A. and Muir, W. W. 2003. Minimum alveolar concentration measures of central nervous system activation in cats anesthetized with isoflurane. Am. J. Vet. Res. 64: 15281533. [Medline] [CrossRef]

16. Mutoh, T., Nishimura, R., Kim, H. Y., Matsunaga, S. and Sasaki, N. 1997. Cardiopulmonary effects of sevoflurane, compared with halothane, enflurane, and isoflurane, in dogs. Am. J. Vet. Res. 58: 885-890. [Medline]

17. Nakajima, R., Nakajima, Y. and Ikeda, K. 1993. Minimum alveolar concentration of sevoflurane in elderly patients. $\mathrm{Br}$. $J$. Anaesth. 70: 273-275. [Medline] [CrossRef]

18. Ohta, M., Oku, K., Yamanaka, T. and Mizuno, Y. 2000. Anesthetic management with sevoflurane and oxygen for orthopedic surgeries in racehorses. J. Vet. Med. Sci. 62: 1017-1020. [Medline] [CrossRef]

19. Rampil, I. J., Mason, P. and Singh, H. 1993. Anesthetic po- 
tency (MAC) is independent of forebrain structures in the rat. Anesthesiology 78: 707-712. [Medline] [CrossRef]

20. Roizen, M. F., Horrigan, R. W. and Frazer, B. M. 1981. Anesthetic doses blocking Adrenergic (stress) and cardiovascular responses to incision-MAC BAR. Anesthesiology 54: 390398. [Medline] [CrossRef]

21. Schmeling, W. T., Ganjoo, P., Staunton, M., Drexler, C. and Farber, N. E. 1999. Pretreatment with dexmedetomidine: altered indices of anesthetic depth for halothane in neuraxis of cats. Anesth. Analg. 88: 625-632. [Medline] [CrossRef]

22. Stanski, D. R. 2000. Monitoring depth of anesthesia. pp. 10871116. In: Anesthesia, 5th ed. (Miller, R. D., ed.), Churchill Livingstone Inc., New York.

23. Steffey, E. P. and Mama, K. R. 2007. Inhalation anesthetics. pp.355-394. In: Lumb and Jones' Veterinary Anethesia. 4th ed. (Tranquilli, W. J., Thurmon, J. C. and Grim, K. A. eds.), Blackwell Publishing, Ames.

24. Ura, T., Higuchi, H., Taoda, M. and Sato, T. 1999. Minimum alveolar concentration of sevoflurane that blocks the adrenergic response to surgical incision in women: MAC-BAR. Eur.
J. Anaesthesiol. 16: 176-181. [Medline]

25. Wilmore, D. W. 2000. Metabolic response to severe surgical illness: overview. World J. Surg. 24: 705-711. [Medline] [CrossRef]

26. Yamashita, K., Iwasaki, Y., Umar, M. A. and Itami, T. 2009. Effect of age on minimum alveolar concentration (MAC) of sevoflurane in dogs. J. Vet. Med. Sci. 71: 1509-1512. [Medline] [CrossRef]

27. Yamashita, K., Muir, W. W. 3rd., Tsubakishita, S., Abrahamsen, E., Lerche, P., Izumisawa, Y. and Kotani, T. 2002. Infusion of guaifenesin, ketamine, and medetomidine in combination with inhalation of sevoflurane versus inhalation of sevoflurane alone for anesthesia of horses. J. Am. Vet. Med. Assoc. 221: 1150-1155. [Medline] [CrossRef]

28. Yamashita, K., Okano, T., Yamashita, M., Umar, M. A., Kushiro, T. and Muir, W. W. 2008. Effects of carprofen and meloxicam with or without butorphanol on the minimum alveolar concentration of sevoflurane in dogs. J. Vet. Med. Sci. 70: 29-35. [Medline] [CrossRef] 